\title{
Skeletal Bone Age Analysis Using Emroi Technique
}

\author{
Dr. Shubhangi D. C ${ }^{1}$, Sweta $^{2}$ \\ ${ }^{1}$ Professor, Department of Computer Science And Engineering, Poojya Doddappa Appa College of Engineering, \\ Gulbarga, Karnataka, India, \\ ${ }^{2}$ Department of Computer Science And Engineering, Poojya Doddappa Appa College of Engineering, Gulbarga,
} Karnataka, India,

\begin{abstract}
Bones are calcified connective tissue forming the major portion of the skeleton of most vertebrates. There are about 206 bones in our body and contains more calcium. Bones begin to develop before birth. From the moment of birth until the time one has grown up, bones go through a set a characteristic changes. Therefore the skeletal maturity, or bone age, can be estimated from radiographs of specific bones in the human body. Children who grow exceptionally slow or fast are often examined by making a radiograph of their left hand and wrist. The aim of this work is to develop a system for skeletal bone age estimation using region of extraction. By analyzing left hand $x$-ray image, the feature extracted are CROI(Carpal ROI), EMROI (Ephiphysial/Metaphysial ROI), using discrete wavelet transformation, ISEF edge detector, energy based segmentation, Jacobi method, cell full and vertex full method. Extracted features are classified using k-mean classifier. Results obtained on a sample of 24 X-rays are discussed. The systems were studied and their performances were compared by various other methods.
\end{abstract}

Keywords - Bone age assessment, CROI, EMROI, Jacobi method, TW2 method.

Accepted Date: 20 June 2013

\section{Introduction}

Bone is dense, semirigid, porous, calcified connective tissue forming the major portion of the skeleton of most vertebrates. It consists of a dense organic matrix and an inorganic, mineral component. There are more than 200 different bones in the human body. Bones are rigid organs that constitute part of the endoskeleton of vertebrates. As a person grows from fetal life through childhood, puberty, and finishes growth as a young adult, the bones of the skeleton change in size and shape. These changes can be seen by $\mathrm{x}$-ray. The "bone age" is the average age at which human reach this stage of bone maturation. A human current height and bone age can be used to predict adult height in pediatric radiology [1]. Bones, such as those in the fingers and wrist, contain "growing zones" at both ends called growth plates. These plates consist of special cells responsible for the bones' growth in length. Growth plates are easy to spot on an X-ray because they're softer and contain less mineral, making them appear darker on an X-ray image than the rest of the bone. A difference between bone age with chronological age [2] might indicate a growth problem. In our proposed method bone age is determined by detailed morphological analyses of left hand $\mathrm{x}$-ray, using discrete wavelet transformation or image transformation, energy based segmentation, Jacobi method, EMROI and CROI for feature extraction, ISEF edge detector and k-mean classifier.

\section{Related Work}

Bone is the substance that forms the skeleton of the body. It serves as a storage area for calcium, playing a large role in calcium balance in the blood. Bones are rigid organs that constitute part of the endoskeleton of vertebrates. Bone tissue is a type of dense connective tissue. Bones come in a variety of shapes and have a complex internal and external structure. The epiphysis [1] is the rounded end of a long bone, at its joint with adjacent bone(s). Between the epiphysis and diaphysis lies the metaphysis, including the epiphyseal plate. At the joint, the epiphysis is covered with articular cartilage; below that covering is a zone similar to the epiphyseal plate, known as subchondral bone. 


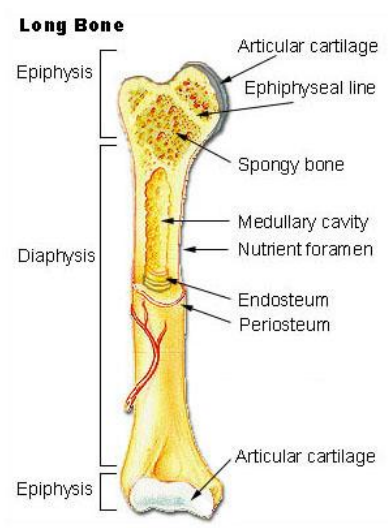

Fig 1: Structure of bone

The diaphysis is the main or midsection of a long bone as shown in "Fig 1". It is made up of cortical bone and usually contains bone marrow and adipose tissue. It's a middle tubular part composed of compact bone which surrounds a central marrow cavity which contains red or yellow marrow. In diaphysis, primary ossification occurs. The metaphysis is the wider portion of a long bone adjacent to the epiphyseal plate. This is the part of the bone that grows during childhood; as it grows, it ossifies near the diaphysis and the epiphyses. At roughly 18 to 25 years of age, the metaphysis stops growing altogether and completely ossifies into solid bone.

Carpus [3] is anatomical assembly connecting the hand to forearm. In human anatomy, the main role of the carpus is to facilitate effective positioning of the hand and powerful use of the extensors and flexors of the forearm, but the mobility of individual carpal bones increase the freedom of movements at the wrist. The carpus is the sole cluster of bones in the wrist between the radius and ulna and the metacarpus as shown in "Fig 2". The bones of the carpus do not belong to individual fingers. The carpal bones allow the wrist to move and rotate vertically.

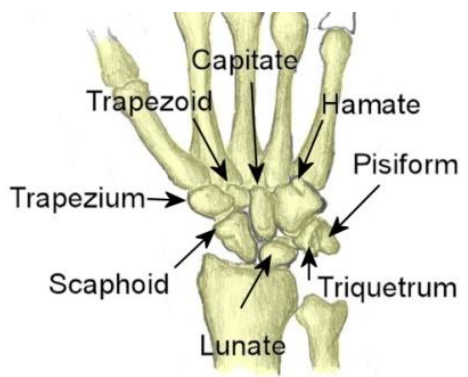

Fig 2 : Structure of carpal bone

The carpal bones are ossified endochondrally (from within the cartilage) and the ossific centers appear only after birth. The formation of these centers roughly follows a chronological spiral pattern starting in the capitate and hamate during the first year of life. The ulnar bones are then ossified before the radial bones, while the sesamoid pisiform arises in the tendon of the flexor carpi ulnaris after more than ten years.

TABLE I : Appearance of ossification centers of carpal bones.

\begin{tabular}{|c|c|c|}
\hline Bone & Average & Variation \\
\hline Capitate & 2.5 months & $1-6$ months \\
\hline Hamate & $4-5.5$ months & $1-7$ months \\
\hline Triquetrum & 2 years & 5 months to 3 years \\
\hline Lunate & 5 years & $2-5.5$ years \\
\hline Trapezium & 6 years & $4-8$ years \\
\hline Trapezoid & 6 years & $4-8$ years \\
\hline Scaphoid & 6 years & $4-7$ years \\
\hline Pisiform & 12 years & $8-12$ years \\
\hline
\end{tabular}

The bone age study can help evaluate how fast or slowly a skeleton is maturing, which can help doctors diagnose conditions that delay or accelerate physical growth and development. Bone age indicate diseases that affect the levels of hormones involved in growth, such as growth hormone deficiency, hypothyroidism, precocious puberty, and adrenal gland disorders, genetic growth disorders [3], such as Turner syndrome (TS). The most commonly used method is based on a single $\mathrm{x}$-ray of the left hand, fingers, and wrist. A hand is easily $\mathrm{x}$-rayed with minimal radiation and shows many bones in a single view. The bones in the $\mathrm{x}$-ray are 
compared to the bones of a standard atlas, usually "Greulich and Pyle" [9][10]. Pal and King used fuzzy functions [4] along with the successive use of contrast intensifier to isolate the regions in the property plane, which could be used for further feature extraction from the X-ray films. (CASAS) system [4] is based on nine prototype images for each bone, representing the nine stages of maturity. Dynamic thresholding technique [4] is used to differentiate between the bones and the soft tissue. The changes in size and shape [5] of bones can be seen by x-ray.BAA is universally used due to its simplicity, minimal radiation exposure, and the availability of multiple ossification centers for evaluation of maturity [6]. The DGHT is a method which has been successfully applied to object localization in medical images. The method combines the Generalized Hough Transform (GHT) with the Discriminative Model Combination (DMC) [7]. A fuzzy methodology is used to translate the natural language descriptions of the TW3 method [8] for bone age assessment into an automatic classifier. The classifier is built upon a modified version of a fuzzy ID3 decision tree. No large data records are needed to train the classifier, i.e., to find out the classification rules, since the classifier is built upon rules given by the TW3 method. A computerized BAE system based on the analysis of carpal features and to evaluate the value of BAE by neural networks [11]. The registration method [12] is based on registering human left hand radiographs for the purpose of bone age determination. The (ACR) k-mean clustering method [13] begins with bands separations into vertical and horizontal direction. Next, the pixels of each section are clustered and performed with GLCM texture analysis. At last, all the sections will be reconstructed based on the texture analysis. Hand Bone Segmentation [14] is done to extract phalangeal and carpal feature from human hand. Statistical models [15] of bone shape and appearance is useful for estimating skeletal maturity.

The methods for skeletal bone age estimation are the Greulich \& Pyle (GP) [5] method and the Tanner \& Whitehouse (TW) [6] method. In GP method, a left-hand wrist radiograph is compared with a series of radiographs grouped in the atlas according to age and sex.TW method uses a detailed analysis of each individual bone, assigning it to one of eight classes reflecting its development stage for bone age. The TW3 [8] method is a commonly accepted procedure in which the guidelines to analyze each bone are described using words (natural language descriptions), sometimes in a vague way. In addition, one particular bone may show features belonging to different stages or a particular bone shape could be classifiable into two possible predefined labels of the same feature. A flexible classification method is needed to manage these sources of ambiguity. Fuzzy logic is known to be a very flexible tool in classification problems where imprecise knowledge or not-welldefined features have to be used.

\section{System Design}

The proposed system contains the following modules as shown in "Fig 3". Image Preprocessing is done to remove noise and eliminate irrelevant, visually unnecessary information and to crop the image, if both hands are present in same x-ray image.

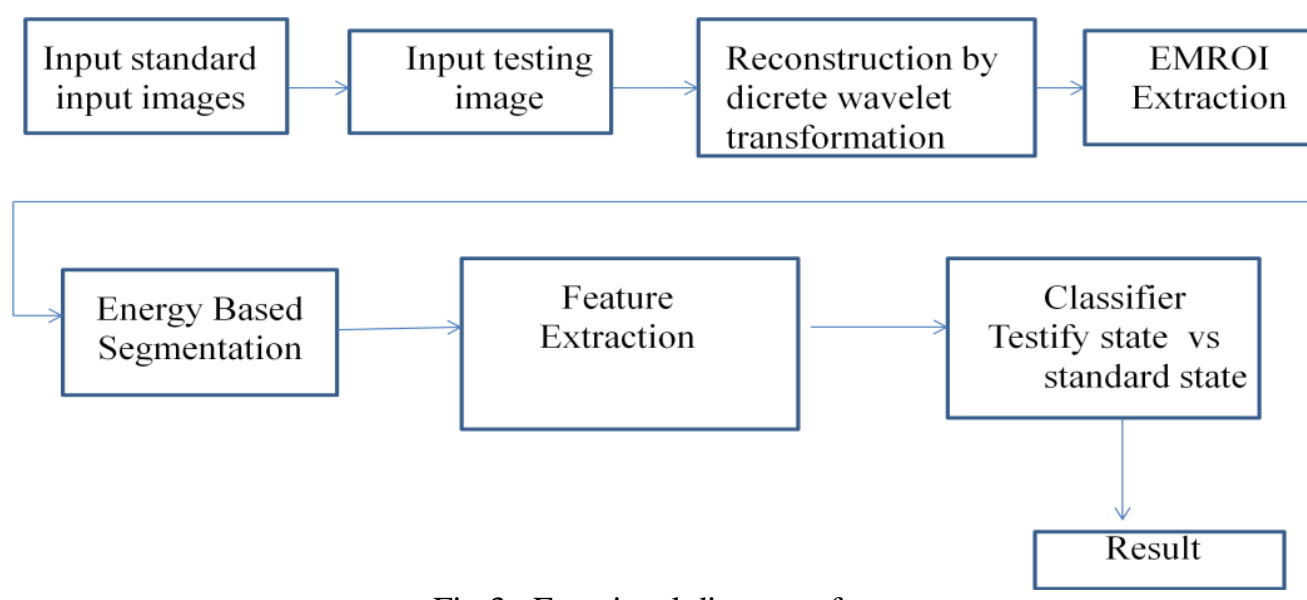

Fig 3 : Functional diagram of system 

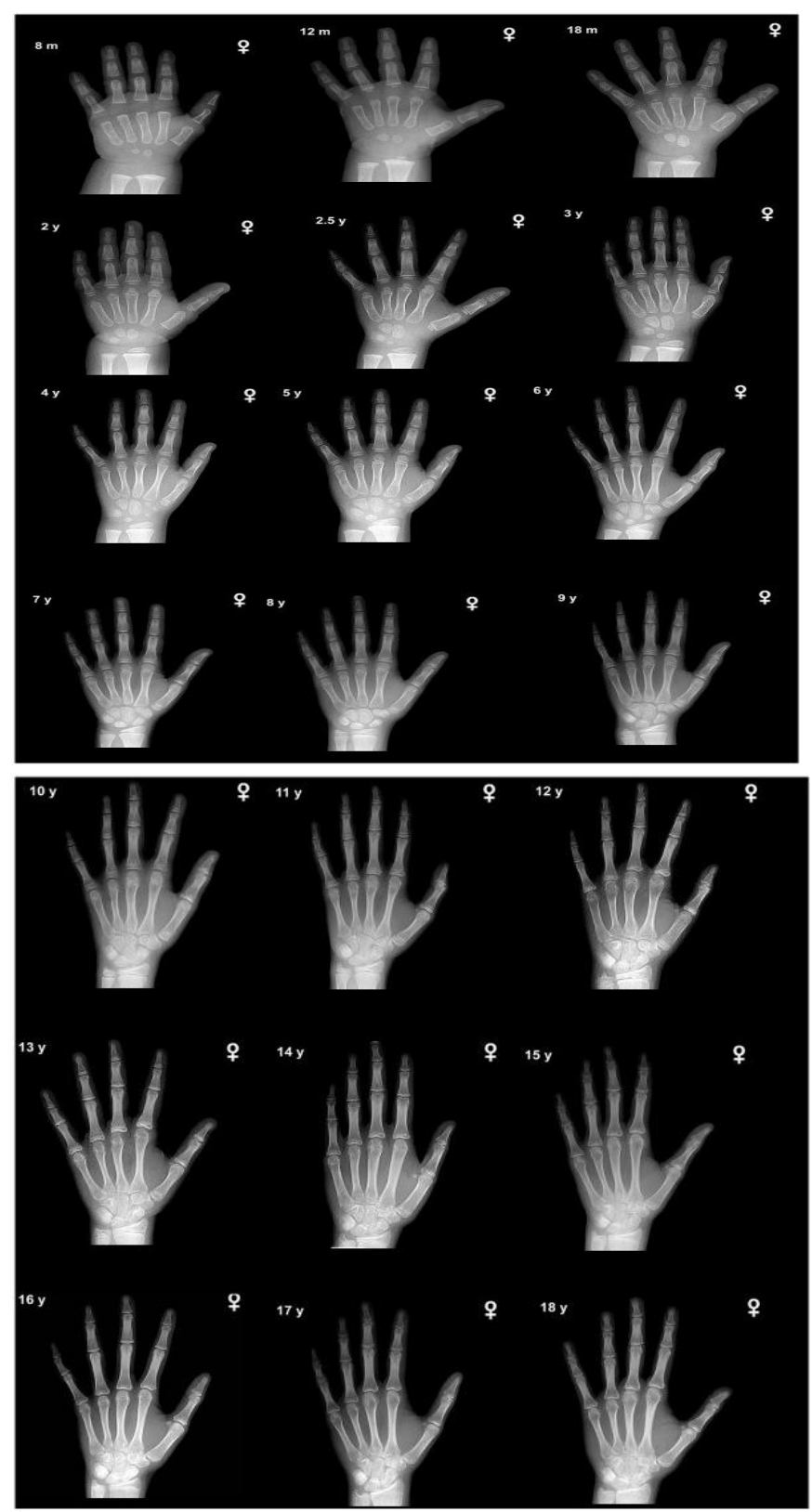

Fig 4: Standard input image set

The input for preprocessing is the standard set of images as shown in "Fig 4". The feature extraction phase starts with testing input image. Image transformation is a function that takes an image as its input and produces an image as its output depending on the transformation chosen, the input and output images may appear entirely different and have different interpretation. The available methods are Fourier transformation, principal component analysis, various spatial filters. For the proposed system we used the discrete wavelength transformation. The DWT of a signal $x$ is calculated by passing it through a series of filters. First the samples are passed through a low pass filter with impulse response $g$ resulting in a convolution of the two :

$$
y[n]=(x * g)[n]=\sum_{k=-\infty}^{\infty} x[k] g[n-k] \quad(1)
$$

A discrete wavelet transform (DWT) is any wavelet transform for which the wavelets are discretely sampled. The transform of a signal is just another form of representing the signal. The Wavelet Transform provides a time-frequency representation of the signal (1). The wavelet transforms captures both frequency and location information. Image segmentation is the process of partitioning a digital image into multiple segments. Image segmentation is typically used to locate objects and boundaries in images. More precisely, image segmentation is the process of assigning a label to every pixel in an image such that pixels with the same label share certain visual characteristics. The result of image segmentation is a set of segments that collectively cover the entire image, or a set of contours extracted from the image. The Energy based segmentation introduces the energy-based segmentation approach wherein the energy of reconstructed surface(s) is directly used to segment the data. For the proposed system Jacobi method is used for first time for bone age 
evaluation along with cell full and vertex full method. Jacobi method is an algorithm used for determining the solutions of a proposed system of linear equations with largest absolute values in each row and column dominated by the diagonal element. Each diagonal element is solved (2) for, and an approximate value plugged in. The process is then iterated until it converges.

The solution is then obtained iteratively via:

The element-based formula is thus:

$$
x^{(k+1)}=D^{-1}\left(b-R x^{(k)}\right)
$$

$$
x_{i}^{(k+1)}=\frac{1}{a_{i i}}\left(b_{i}-\sum_{j \neq i} a_{i j} x_{j}^{(k)}\right), i=1,2, \ldots, n
$$

Note that the computation of $x_{i}^{(k+1)}$ requires each element in $x^{(k)}$ except itself. Unlike the Gauss-Seidel method, it can't overwrite $x_{i}^{(k)}$ with $x_{i}^{(k+1)}$ (3), as that value will be needed by the rest of the computation. The minimum amount of storage is two vectors of size $n$. Feature extracted are the edge detection of hand and wrist, carpal region, epiphysial/metaphysical region, morphology of epiphysial, metaphysical along with carpal bones, shape and distance between object as in "Fig 5".

Morphology here deals with study of bone form and their specific structural features. The metacarpals are more readily described than the phalanges. Because metacarpals are asymmetrical in their morphology which allows them to be easily distinguished from each other. The lengths of the metacarpals and phalanges of the human hand can be used to estimate the length of a long bone which in turn used to determine the bone age.

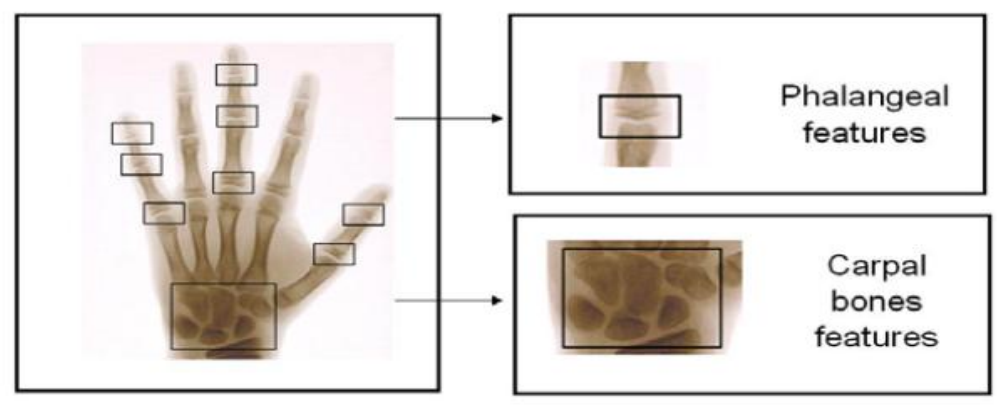

Fig 5: Emrois and Crois

Edge detection is a fundamental of low-level image processing and good edges are necessary for higher level processing. Different edge detection techniques are the marr-hildreth edge detector, the canny edge detector, the local threshold and boolean function based edge detection color edge detection using Euclidean distance and vector angle color edge detection using the canny operator. The ISEF is a very high quality of edge detector on images with high frequency uniform noise, which computes the difference of the exponentially weighted means of each half window. Morphological boundary extraction uses some simple set operations to outline edges on the boundaries of objects in an image. Edge detection is a well-developed field on its own within image processing. Region boundaries and edges are closely related, since there is often a sharp adjustment in intensity at the region boundaries. Edge detection techniques have therefore been used as the base of another segmentation technique. The edges identified by edge detection are often disconnected. To segment an object from an image however, one needs closed region boundaries. The desired edges are the boundaries between such objects. Segmentation methods can also be applied to edges obtained from edge detectors. The proposed system makes use of k-mean classifier. This classifier used to perform classification, or clustering, in a group of images. This is most commonly applied to land cover classification using multi-spectral, remotely sensed images. The algorithm starts by randomly locating $k$ clusters in spectral space. Each pixel in the input image group are then assigned to the nearest cluster centre and the cluster centre locations are moved to the average of their class values. This process is then repeated until a stopping condition is met. The stopping condition may either be a maximum number of iterations or a tolerance threshold which designates the smallest possible distance to move cluster centers before stopping the iterative process. Next, the input images are compared with standard images and bone age is obtained via result phase. And the process is repeated for different set of images. 


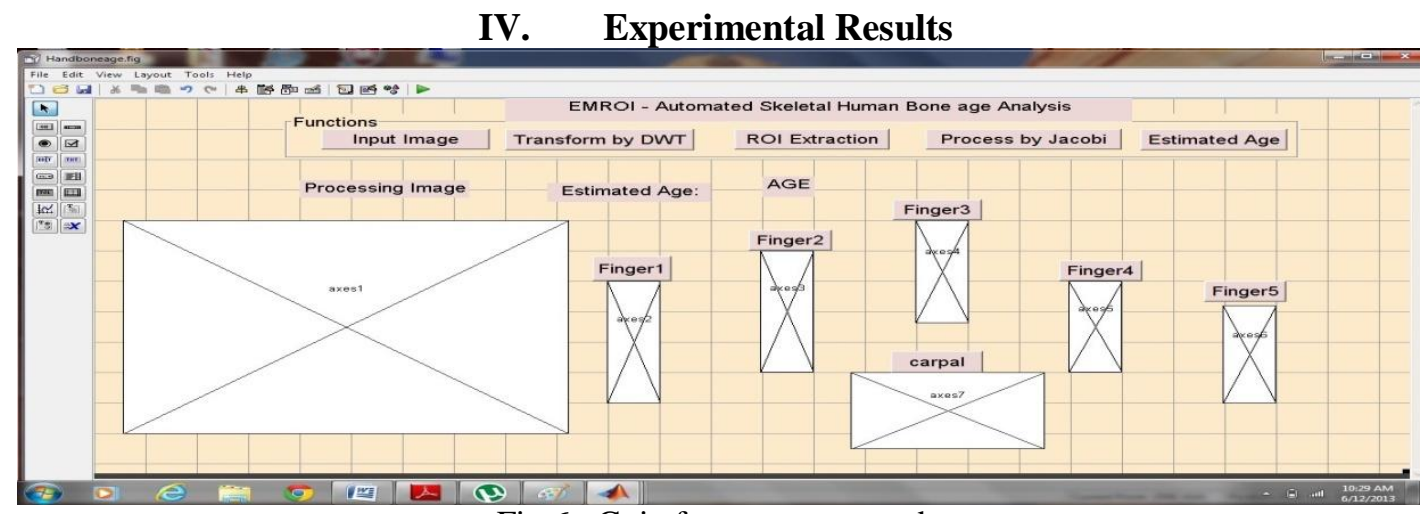

Fig 6 : Gui of system proposed.

Skeletal hand image is given as input, which is transformed using discrete wavelength transformation.

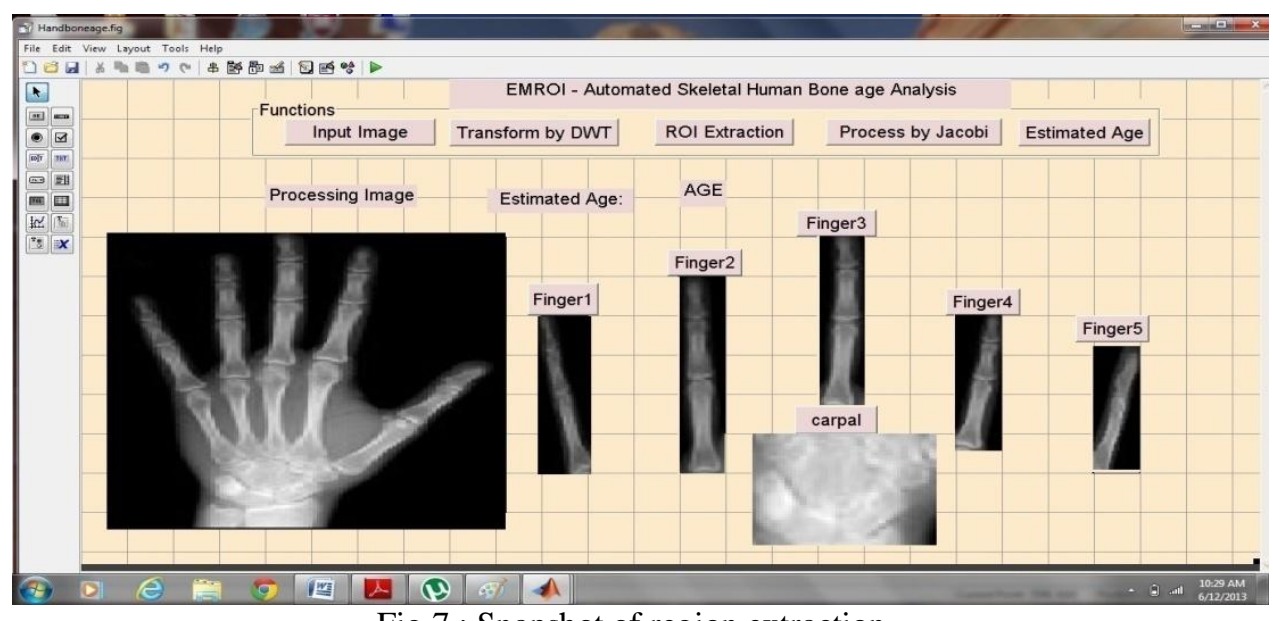

Fig 7 : Snapshot of region extraction

Pattern based ROI extraction is done and feature extracted are phalangeal features and carpal bones features in hand.

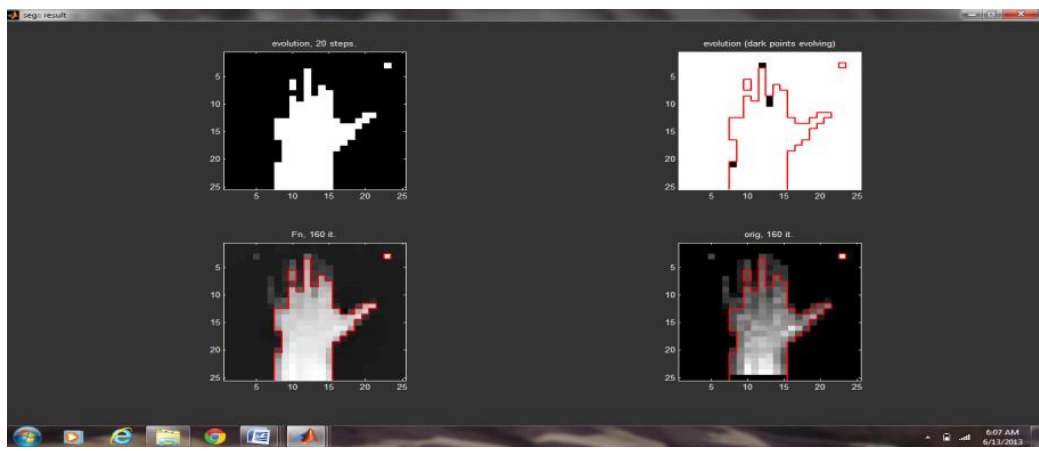

Fig 8 : Snapshot of vertex full for bone age determination.

Vertex full with Jacobi method is used for extraction.

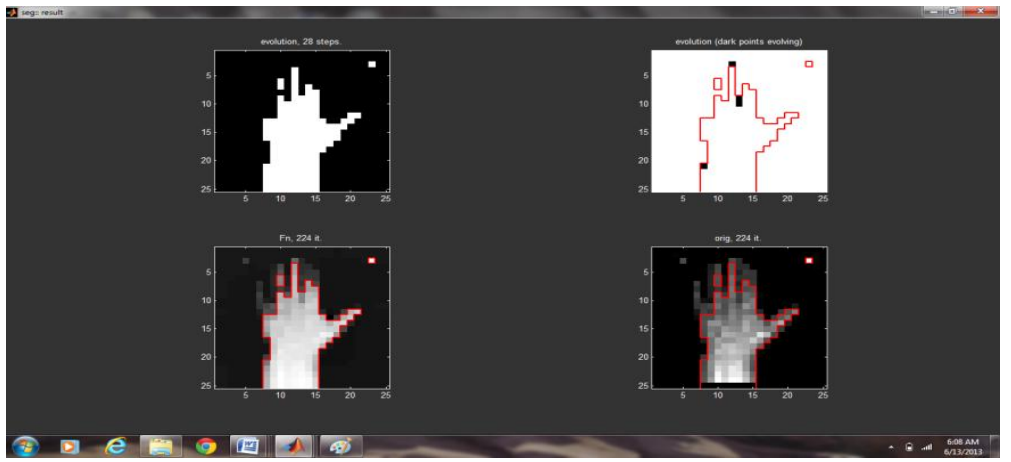

Fig 9 : Snapshot of cell full for bone age determination.

Cell full with Jacobi method is used for extraction. 


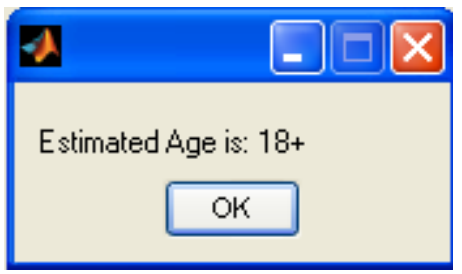

Fig 10 : Snapshot of output

TABLE II : Age determined on different image set

\begin{tabular}{|c|c|c|c|c|c|c|}
\hline \multirow{2}{*}{ S.no } & Image set & \multicolumn{4}{|c|}{ Age determined } & \multirow{2}{*}{ Undetermined } \\
\cline { 3 - 6 } & & $1-5$ & $5-8$ & $8-15$ & $15-18$ & \\
\hline 1 & 50 & 11 & 8 & 12 & 16 & 3 \\
\hline 2 & 100 & 20 & 22 & 24 & 27 & 7 \\
\hline 3 & 250 & 58 & 60 & 53 & 70 & 9 \\
\hline 4 & 500 & 123 & 106 & 156 & 100 & 15 \\
\hline
\end{tabular}

TABLE III : Performance accuracy

\begin{tabular}{|c|c|c|}
\hline S.no & Age & Proposed system accuracy \\
\hline 1 & 8 months to 2 years & $90 \%$ \\
\hline 2 & 2 to 5 years & $91 \%$ \\
\hline 3 & 5 to 8 years & $93 \%$ \\
\hline 4 & 8 to 12 years & $94 \%$ \\
\hline 5 & 12 to 15 years & $95 \%$ \\
\hline 6 & 15 to 18 years & $97 \%$ \\
\hline
\end{tabular}

The proposed approach has been tested on 24 standard x-ray images and various image sets. We used for bone age assessment comparing standard images with input images. Region of extraction is done on carpal bones, later on Epiphyseal/Metaphyseal Region Of Interest. We found that using carpal bones extraction bone age assessment found $92-98 \%$ for $\mathrm{x}$-ray images. Concerning classification, the average matching Percentage between the obtained results and the radiologist's evaluations is about $87 \%$. The main advantage of the proposed system is that it presents a high success rate of $95 \%$ with standard set of images, with removal of improper set of images.

\section{Conclusion}

Skeletal maturity can be assessed in a number of ways. From the X-ray images as input for the proposed system, the bone age can be estimated. From this estimate, together with other pieces of information such as the calendar age, sex and height of the child and possibly information about the parents, the adult height can be predicted. A big discrepancy between the calendar age and the bone age can indicate an atypical skeletal development. In many cases the decision whether to treat a child with growth hormones depends on the outcome of bone age estimation. We used EMROI, CROI, Jacobi method with morphological features in proposed system. CROI based assessment investigates the size and shape of the carpal bones. In the newborn, the carpal bones are nothing more than well defined dots, which grow bigger almost uniformly as the subject ages. This is the reason that, in contrast to the EMROI approach, the CROI approach is more accurate in assessing skeletal chronological maturity. Bone age assessment can be done on IR Imaging. Hence we found this proposed system gives higher performance with great accuracy. This bone age assessment system offers a reliable, simpler and more efficient alternative to current methods, for use by residents, fellows, radiologists, and pediatric orthopaedic surgeons.

\section{References}

[1] D. Giordano, R. Leonardi, F. Maiorana, G. Scarciofalo, and C. Spampinato: "Epiphysis and Metaphysis Extraction and Classification by Adaptive Thresholding and DoG Filtering for Automated Skeletal Bone Age Analysis" Proceedings of the 29th Annual International Conference of the IEEE EMBS Cité Internationale, Lyon, France August 23-26, 2007.

[2] P. Thangam, K. Thanushkodi ,T. V. Mahendiran: "Comparative Study of Skeletal Bone Age Assessment Approaches using Partitioning Technique"International Journal of Computer Applications (0975 - 8887) Volume 45- No.18, May 2012.

[3] N. Olarte L, A. Rubiano F, A. Mejía F: “Comparison of Valuation Techniques for Bone Age Assessment" World Academy of Science, Engineering and Technology 682012.

[4] P.Thangam, T.V.Mahendiran and K. Thanushkodi: "Skeletal Bone Age Assessment - Research Directions" Review Article Journal of Engineering Science and Technology Review 5 (1) (2012) 90 - 96.

[5] P. Thangam,K. Thanushkodi,\&T. V. Mahendiran: "Computerized Convex Hull Method of Skeletal Bone Age Assessment from Carpal Bones” European Journal of Scientific Research ISSN 1450-216X Vol.70 No.3 (2012), pp. 334-344. 
[6] P.Thangam, K.Thanushkodi, T.V.Mahendiran: "Efficient Feature Analysis of Radiographs in Bone Age Assessment" International Journal of Computer Applications in Engineering Sciences[VOL I,ISSUE II,JUNE 2011]

[7] Markus Brunk, Heike Ruppertshofen, Sarah Schmidt, Peter Beyerlein, Hauke Schramm: "Bone Age Classification Using the Discriminative Generalized Hough Transform".

[8] Santiago Aja-Fernfandez, Rodrigo de Luis-Garc_1a, Miguel _ Angel Mart_n-Fern_andez, and Carlos Alberola-L_opez: "A computational TW3 classifier for skeletal maturity assessment. A Computing with Words approach" Journal of Biomedical Informatics 37 (2004) 99-107.

[9] Daniela Giordano, Member, IEEE, Concetto Spampinato, Giacomo Scarciofalo and Rosalia Leonardi: "An Automatic System for Skeletal Bone Age Measurement by Robust Processing of Carpal and Epiphysial/Metaphysial Bones".

[10] Rick R. van Rijn \&Maarten H. Lequin \& Hans Henrik Thodberg: "Automatic determination of Greulich and Pyle bone age in healthy Dutch children”Pediatr Radiol (2009) 39:591-597 DOI 10.1007/s00247-008-1090-8.

[11] Chi-Wen Hsieh, Tai-Lang Jong, Yi-Hong Chou and Chui-Mei Tiu: "Computerized geometric features of carpal bone for bone age estimation" Chinese Medical Journal 2007;120(9):767-770 767.

[12] Miguel A. Martin-Fernandez, Marcos Martin-Fernandez, Carlos Alberola-Lopez: “Automatic bone age assessment: a registration approach"ETSI Telecomunication, Universidad de Valladolid Campus Miguel Delibes s/n, 47011 Valladolid, Spain.

[13] Hum Yan Chai, Lai Khin Wee, Tan Tian Swee and Sh-Hussain Salleh: "Adaptive Crossed Reconstructed (ACR) K-mean Clustering Segmentation for Computer aided Bone Age Assessment System” International Journal of Mathematical Models And Methods In Applied Sciences.

[14] Tran Thi My Hue, Jin Young Kim, Mamatov Fahriddin: "Hand Bone Radiograph Image Segmentation With ROI Merging” Recent Researches in Mathematical Methods in Electrical Engineering and Computer Science.

[15] Steve A. Adeshina, Timothy F. Cootesyand Judith E. Adams: "Evaluating different structures for predicting skeletal maturity using statistical appearance models" Imaging Science and Biomedical Engineering, The University of Manchester, Manchester UK. 